\title{
Zeeman splitting of conduction band in HgTe quantum wells near the Dirac point
}

\author{
G. M. Minkov, ${ }^{1}$ O. E. Rut,${ }^{1}$ A. A. Sherstobitov, ${ }^{1,2}$ S. A. Dvoretski, ${ }^{3}$ and N. N. Mikhailov ${ }^{3,4}$ \\ ${ }^{1}$ Institute of Natural Sciences, Ural Federal University, 620002 Ekaterinburg, Russia \\ ${ }^{2}$ M. N. Miheev Institute of Metal Physics of Ural Branch of Russian Academy of Sciences, 620137 Ekaterinburg, Russia \\ ${ }^{3}$ Institute of Semiconductor Physics RAS, 630090 Novosibirsk, Russia \\ ${ }^{4}$ Novosibirsk State University, Novosibirsk 630090, Russia
}

(Dated: September 6, 2018)

\begin{abstract}
The Zeeman splitting of the conduction band in the HgTe quantum wells both with normal and inverted spectrum has been studied experimentally in a wide electron density range. The simultaneous analysis of the $\mathrm{SdH}$ oscillations in low magnetic fields at different tilt angles and of the shape of the oscillations in moderate magnetic fields gives a possibility to find the ratio of the Zeeman splitting to the orbital one and anisotropy of $g$-factor. It is shown that the ratios of the Zeeman splitting to the orbital one are close to each other for both types of structures, with a normal and inverted spectrum and they are close enough to the values calculated within $k P$ method. In contrast, the values of $g$-factor anisotropy in the structures with normal and inverted spectra is strongly different and for both cases differs significantly from the calculated ones. We believe that such disagreement with calculations is a result of the interface inversion asymmetry in the $\mathrm{HgTe}$ quantum well, which is not taken into account in the $k P$ calculations.
\end{abstract}

\section{INTRODUCTION}

A HgTe/CdTe quantum well is a system where the Dirac fermions appear only in a single valley, at the $\Gamma$ point of the Brillouin zone, unlike graphene where there are two valleys of the Dirac fermions with a strong intervalley scattering. The energies of spatially quantized subbands at the quasimomentum $k=0$ and energy spectrum $E(k)$ for different widths of the quantum well $(d)$ were calculated within $k P$ method in numerous papers [1 5 ]. As seen from Fig. 1. various types of energy spectrum are realized upon increasing the HgTe quantum-well width; namely, "normal", when $d$ is less than a critical width $d_{c} \simeq 6.3 \mathrm{~nm}$, Dirac-like at small quasimomenta for $d=$ $d_{c}$, inverted when $d>d_{c}$, and finally, semimetallic when $d>14-16 \mathrm{~nm}$. To interpret experimental data, these calculations of the energy spectrum are used practically always. They well describe the width dependence of the energies of both electron and hole subbands at $k=0$ and the energy dependence of the electron effective mass $\left(m_{e}\right)$.

However, quite a lot of differences between the experimental data and the results of these calculations on the energy spectrum of the carriers have been accumulated to date. First of all, they refer to the spectrum of the valence band. The hole effective mass $\left(m_{h}\right)$ at $d \approx 20 \mathrm{~nm}$ within the wide hole density range $p=(1-4) \times 10^{11} \mathrm{~cm}^{-2}$ is substantially less than the calculated one: $m_{h} \simeq(0.15-0.3) m_{0}[\underline{6}, 6]$ instead of $(0.5-0.6) m_{0}[5]$. The top of the valence band in the nominally symmetric structures with $d \approx d_{c}(d=5.5-7 \mathrm{~nm})$ was found to be very strongly split by spin-orbit (SO) interaction [8]. Therewith, the SO splitting of the conduction band in the same structures does not reveal itself [9]. It is surprising that such SO splitting is observed in structures both with inverted and normal spectrum despite the fact that at $d<d_{c}$ and $d>d_{c}$ the conduction band is formed from different terms (see Fig. 11). At $d<d_{c}$, the conduction band is formed from electron states and states of light hole, while at $d>d_{c}$, it is formed from heavy-hole states. Such SO splitting was not described by Byckov-Rashba effect taken into account within $k P$ method. It was assumed 9] that such a surprising behavior of SO splitting is a result of the interface inversion asymmetry (IIA) in the HgTe quantum well, which was not taken into account in $k P$ calculations in [1 [5].

The question arises: how other spin-dependent effects, for example, the Zeeman splitting, depend on the spectrum type - normal or inverted. We found only two papers where the Zeeman splitting of electron spectrum was measured in the $\mathrm{HgTe}$ quantum wells with the width $d$ which is more or less close to $d_{c}$ [10, 11]. In Ref. [10], the Zeeman splitting was determined in a structure with normal spectrum, $d=6.1 \mathrm{~nm}$, at very large electron density $n=1.4 \times 10^{12} \mathrm{~cm}^{-2}$. In Ref. [11], it was determined in a structure with inverted spectrum with $d=9 \mathrm{~nm}$ that is noticeably larger than $d_{c}$, at $n=6.59 \times 10^{11} \mathrm{~cm}^{-2}$.

So, up to now a systematic study of the Zeeman splitting and a comparison of it with theoretical calculations are absent. In this paper, we present the results of the investigation of the Shubnikov-de Haas $(\mathrm{SdH})$ oscillations in tilt magnetic fields in the $\mathrm{HgTe}$ quantum wells with normal and inverted spectra. To find the ratio of the Zeeman splitting to the orbital one, we have used a modified coincidence method which consists in measuring the angle dependence of amplitudes of the $\mathrm{SdH}$ oscillations in low magnetic fields. The simultaneous analysis of this dependence and the shape of oscillations of $\rho_{x x}$ made it possible to determine both the ratio of the Zeeman splitting to the cyclotron one and the anisotropy of $g$-factor $\left(g_{\|} / g_{\perp}\right)$ over a wide electron-density range, where $g_{\|}$and $g_{\perp}$ are the in-plane and transverse $g$-factor, respectively. 

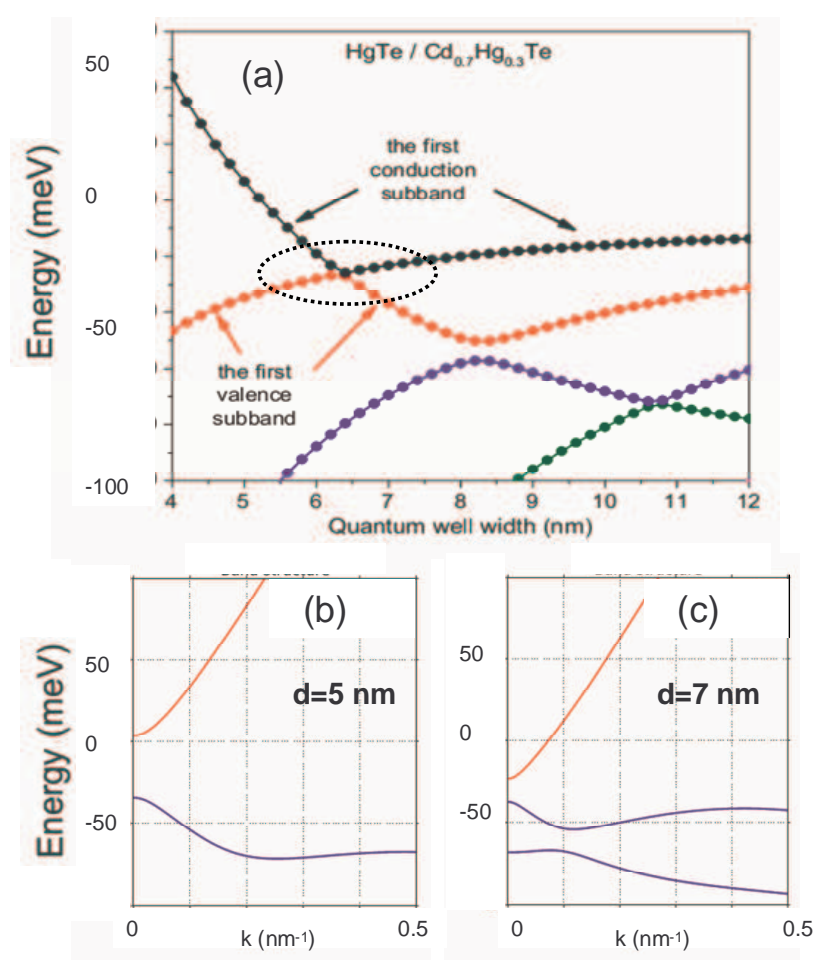

Figure 1. (Color online) (a) - The quantum well width dependence of the subband energies at $k=0$. The dependences $E(k)$ of the conduction and valence bands for $d<d_{c}$ (b) and $d>d_{c}$ (c). The marked area in (a) shows the range of quantum well widths under study.

\section{EXPERIMENT}

Our samples with the HgTe quantum wells were realized on the basis of $\mathrm{HgTe} / \mathrm{Hg}_{1-x} \mathrm{Cd}_{x} \mathrm{Te}(x=0.55-0.65)$ heterostructures grown by the molecular beam epitaxy on a GaAs substrate with the (013) surface orientation [12]. The samples were mesa etched into standard Hall bars of $0.5 \mathrm{~mm}$ width and the distance between the potential probes was $0.5 \mathrm{~mm}$. To change and control the carrier density in the quantum well, the field-effect transistors were fabricated with parylene as an insulator and aluminium as a gate electrode. For each heterostructure, several samples were fabricated and studied. The Zeeman splitting of the conduction band has been obtained from measurements of the $\mathrm{SdH}$ effect in a tilted magnetic field, i.e. we used the so-called coincidence method. This method is based on the fact that the spin splitting, $g \mu_{B} B$, depends on the total magnetic field $(B)$ whereas the orbital splitting of the Landau levels (LLs) in 2D systems, $\hbar \omega_{c}$, is proportional to the component of the magnetic field which is perpendicular to the $2 \mathrm{D}$ plane $\left(B_{\perp}\right)$ : $\hbar \omega_{c}=\left(e \hbar / m_{e}\right) B_{\perp}=\left(e \hbar / m_{e}\right) b B$, where $b \equiv B_{\perp} / B$.

Thus, the ratio of the Zeeman splitting to the orbital one, $g \mu_{B} B / \hbar \omega_{c}$, will change upon varying the tilt angle as $X(b)=g \mu_{B} m_{e} /(e \hbar B b)$. It is clear that there are particular angles $b_{c}$ when $X\left(b_{c}\right)=1 / 2,1,3 / 2 \ldots$ At inte-
Table I. The parameters of heterostructures under study

\begin{tabular}{ccccc}
\hline \hline number & structure & $d(\mathrm{~nm})$ & type & $p, n\left(V_{g}=0\right)\left(\mathrm{cm}^{-2}\right)$ \\
\hline 1 & 1520 & 4.6 & $n$ & $1.5 \times 10^{11}$ \\
2 & 1122 & 5.6 & $p$ & $1.3 \times 10^{11}$ \\
3 & 1023 & 6.5 & $p$ & $1.0 \times 10^{10}$ \\
4 & H725 & 8.3 & $p$ & $5.0 \times 10^{10}$ \\
5 & HT71 & 9.5 & $p$ & $6.0 \times 10^{10}$ \\
\hline \hline
\end{tabular}

ger $X\left(b_{c}\right)$ values, the energies of the LLs with different numbers and opposite spin coincide with each other and the distances between the pairs of such degenerate LLs are equal to $\hbar \omega_{c}$. When $X\left(b_{c}\right)$ is half-integer, the energy distances between nearest LLs are twice as low, $0.5 \hbar \omega_{c}$. As a result, the oscillation periods will differ twice for these cases. Knowing values of $b_{c}$, one can find the ratio $X(1) \equiv X$. For example, when $X\left(b_{c}\right)=1 / 2, X=b_{c} / 2$.

In this paper, we used the modified coincidence method [13 15]. We have measured the oscillations at low magnetic field when: (i) $B_{\perp}$ is significantly less than the field of the onset of the quantum Hall effect (QHE); (ii) amplitude of the oscillation is small so that oscillations of the Fermi energy are negligible; (iii) the $\mathrm{SdH}$ oscillations are spin-unsplit. In this case, the study of the angular dependence of the oscillation amplitude $A(b)$ at a given $B_{\perp}$ value within the entire range of angles (rather than the determination of critical angles) allows one not only to determine the ratio of the Zeeman splitting to the orbital one, but estimate the $g$-factor anisotropy.

To find analytic expression for the tilt-angle dependence of the oscillation amplitude $A(b)$, it is convenient to represent the oscillations as the sum of the contributions from the two series of the Landau spin sublevels. At low magnetic field, when the $\mathrm{SdH}$ oscillations are unsplit, the main contribution to the oscillations of $\rho_{x x}$ comes from the first harmonic, [16] and the well known LifshitsKosevitch (LK) formula for the $\mathrm{SdH}$ oscillations reduces to the following expression

$$
\begin{aligned}
\Delta \rho_{x x}(B, b) & =\rho_{x x}(B, b)-\rho_{x x}(0) \\
& =a_{\uparrow} \cos \left[2 \pi\left(\frac{E_{F}}{\hbar \omega_{c}}+\frac{1}{2}+\frac{X(b)}{2}\right)\right] \\
& +a_{\downarrow} \cos \left[2 \pi\left(\frac{E_{F}}{\hbar \omega_{c}}+\frac{1}{2}-\frac{X(b)}{2}\right)\right] .
\end{aligned}
$$

Here, the factors $a_{\uparrow}$ and $a_{\downarrow}$ depend on the Dingle factor, temperature, and magnetic field. When $a_{\uparrow}=a_{\downarrow}=a$, Eq. (1) is

$$
\Delta \rho_{x x}(B, b)=2 a \cos [\pi X(b)] \cos \left[2 \pi\left(\frac{E_{F}}{\hbar \omega_{c}}+\frac{1}{2}\right)\right] .
$$

Thus, over this magnetic field range, the values of $B_{\perp}$ corresponding to the extremes of $\rho_{x x}$ should not depend on the tilt angle, while the amplitudes of oscillations $A(b)=2 a \cos [\pi X(b)]$ should periodically change with 

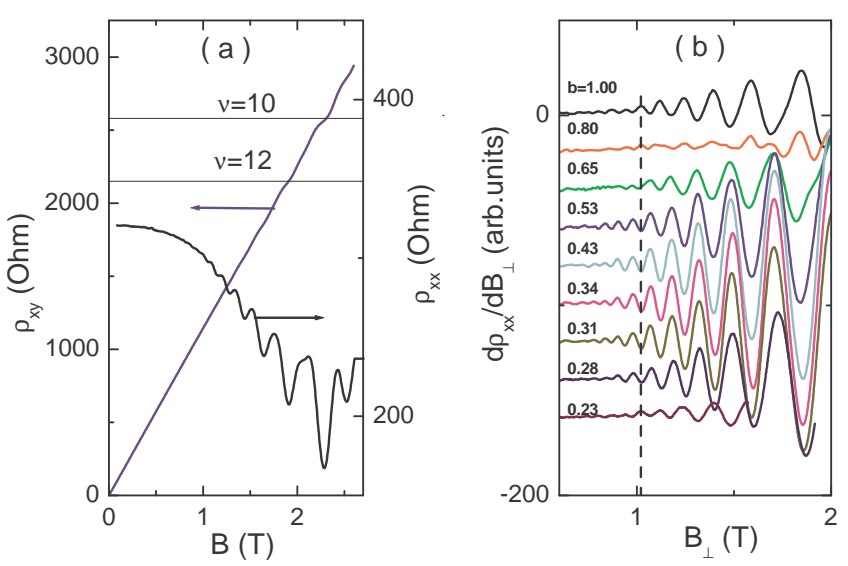

Figure 2. (Color online) (a) The magnetic field dependences of $\rho_{x x}$ and $\rho_{x y}$ at normal magnetic field in structure 1520 at $n=5.45 \times 10^{11} \mathrm{~cm}^{-2} . T=4.2 \mathrm{~K}$. (b) The dependences of $d \rho_{x x} / d B_{\perp}$ on $B_{\perp}$ for different $b$.

$b$ and the angular dependence of the relative amplitude is

$$
\frac{A(b)}{A(1)}=\frac{\cos [\pi X(b)]}{\cos [\pi X(1)]}
$$

\section{RESULTS AND DISCUSSION}

Let us begin our analysis with the results obtained in the structures with a normal spectrum $\left(d<d_{c}\right)$ (see Table I). As an example, consider the results for the structure 1520. Before discussing the oscillations in the tilt magnetic field, it is necessary to estimate the magnetic field range where Eq. (11) is valid for this structure. To this end, let us inspect the magnetic field dependences of $\rho_{x x}$ and $\rho_{x y}$ in the normal field, which are presented in Fig. 2(a) for the electron density $n=5.45 \times 10^{11} \mathrm{~cm}^{-2}$. It is seen that at $B<1.5 \mathrm{~T}$, the amplitude of oscillations of $\rho_{x x}$ is 10 percent less, and the steps in $\rho_{x y}(B)$ (with even filling factors $\nu$ ) appear only at $B>1.5 \mathrm{~T}$; therefore one can neglect the oscillations of the Fermi energy within this range of $B$. The electron density found from the period of oscillations under assumption that the Landau levels are two-fold degenerate, coincides with the Hall density [17]. Thus, at $B<1.5 \mathrm{~T}$, the conditions of applicability of Eq. (1) are met.

Now let us inspect the SdH oscillations in the tilt magnetic field. To remove the monotonic part we plotted in Fig. 2(b) the $d \rho_{x x} / d B_{\perp}$ versus $B_{\perp}$ dependences, measured at different tilt angles. To make it easier to trace the position of oscillations at different angles, we mark the position of one of the maxima $d \rho_{x x} / d B$ at the normal field $B=1.02 \mathrm{~T}$ by a dashed line. It is clearly seen that the positions of extremes of $d \rho_{x x} / d B_{\perp}$ do not change with tilt angle but the maxima are transformed to the minima at $b \simeq 0.65$, and upon further rotation they are

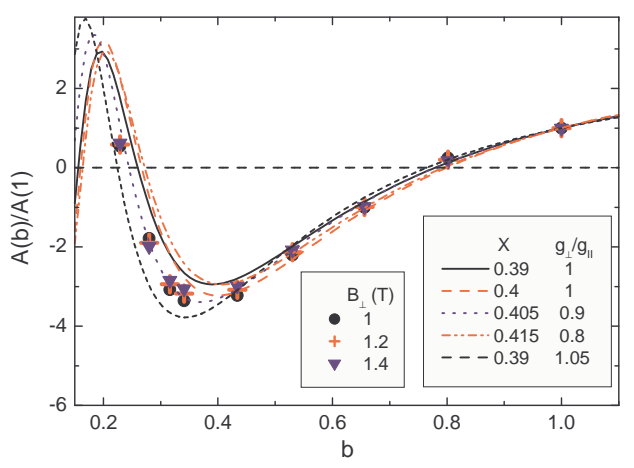

Figure 3. (Color online) The relative oscillations amplitude for the structure H1520 plotted against $b$ at $n=$ $5.45 \times 10^{11} \mathrm{~cm}^{-2}$. Points are experimental data found at $B_{\perp}=1.0,1.2$ and $1.4 \mathrm{~T}$. The inversion of the amplitude sign corresponds to the change of the oscillation phase by $\pi$. Solid and dash lines are the dependences Eq.(3) with $X=0.39$ and 0.4 , respectively. Other lines are the calculated dependences with taking into account $g$-factor anisotropy.

transformed to the maxima again at $b \simeq 0.23$. Note, a noticeable difference was not observed when the parallel component of the field was along or perpendicular to the current.

For the quantitative analysis, the amplitude of oscillations $A\left(B_{\perp}, b\right)$ at a given $b$ was found by fitting of the oscillatory part of $A\left(B_{\perp}, b\right)$ versus $B_{\perp}$ curves to the oscillating function corresponding to the electron density $n=1 / e R_{H}$ measured in the normal field, with the amplitude $A(B)=k_{0} \exp \left(k_{1} B\right)$. The relative amplitudes of the oscillations found in this way $A(b) / A(1)$ as a function of $b$ are plotted for some values of $B_{\perp}$ in Fig. 3. The inversion of the amplitude sign corresponds to the change in the oscillations phase by $\pi$. Note, that $A(b) / A(1)$ does not depend practically on $B_{\perp}$ when the magnetic field is sufficiently small so that Eq. (1) is valid. In the same figure we have shown the dependences which are given by Eq. (3) for some values of the ratio $X(b)=g \mu_{B} B /\left(e \hbar B_{\perp} / m_{e}\right)$. One can see that Eq. (3) well describes the experimental data with $X=0.39 \pm 0.01$. Some deviation at $b<0.3$ can result from the $g$-factor anisotropy. Indeed, taking this possibility into account in simplest form

$$
g(b)=\sqrt{g_{\perp}^{2} b^{2}+g_{\|}^{2}\left(1-b^{2}\right)}
$$

with $g_{\perp} / g_{\|}=0.9$ we obtain the exact coincidence over all range of tilt angles with $X=0.405$ (see dot line in Fig. 3). In Fig. 3, we have plotted also the angular dependences of $A(b) / A(1)$ with close pairs of parameters. This makes it possible to assess how uniquely these parameters are determined. Note that the value of $X<0.5$ is consistent with the fact that the onset of QHE is observed with even numbers [see Fig. 2(a)].

Such data treatment was carried out for other electron densities and all the results for $g \mu_{B} B / \hbar \omega_{c}$ versus 

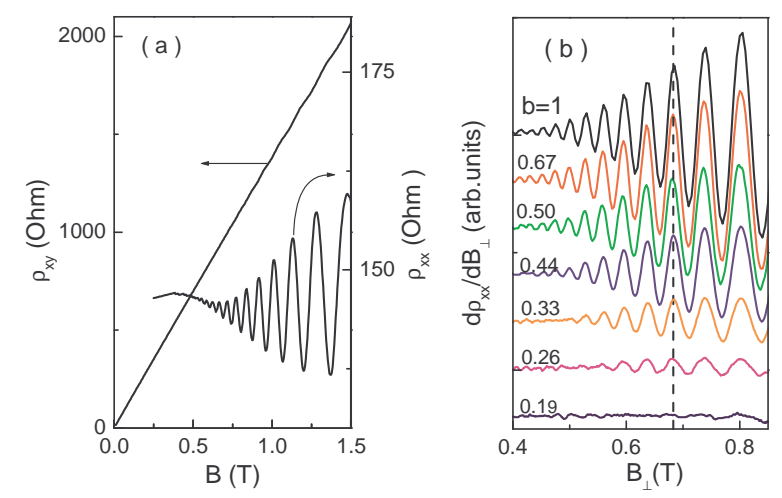

Figure 4. (Color online) (a) The magnetic field dependences of $\rho_{x x}$ and $\rho_{x y}$ at normal magnetic field for the structure $1023\left(d<d_{c}\right)$ at $n=4.6 \times 10^{11} \mathrm{~cm}^{-2}$. $T=4.2 \mathrm{~K}$. (b) The dependences of the derivatives $d \rho_{x x} / d B_{\perp}$ on $B_{\perp}$ for different $b$.

the electron density are plotted in Fig. 7 together with the results obtained for another structure with $d<d_{c}$, 1122 (see Table \).

Before comparing these results with the theoretical dependences of $g \mu_{B} B / \hbar \omega_{c}$ on the electron density, let us consider the data for structures with inverted spectrum $\left(d>d_{c}\right)$.

As an example, in Fig. 4, we have presented the data for structure 1023 at $n=4.6 \times 10^{11} \mathrm{~cm}^{-2}$. One can see that at $B<0.8 \mathrm{~T}$, the oscillations of $\rho_{x y}$ are practically absent and the spin splitting of the oscillations of $\rho_{x x}$ is not observed. So, at $B<0.8 \mathrm{~T}$, the conditions for applicability of Eq. (11) are met. The derivatives $d \rho_{x x} / d B_{\perp}$, measured at different tilt angles as a function of $B_{\perp}$ are presented in Fig. 4(b) for different $b$. For clarity, we have plotted the dashed line at $B_{\perp}=0.68 \mathrm{~T}$ which corresponds to the position of one of maxima. It is clearly seen that with the tilt angle increase, the positions of the extremes of $d \rho_{x x} / d B$ in $B_{\perp}$, similarly to in structures with $d<d_{c}$ (see. Fig. 2 $\mathrm{b}$ ), does not change but the amplitude of the oscillations decreases significantly slower than in the structure with $d<d_{c}$, and the inversion of the oscillations phase is not observed up to $b=0.19$ [compare Fig. 2(b) and Fig. 4(b)].

The dependence of the amplitude of oscillations on $b$ together with the calculated dependence, Eq. (3), with the isotropic $g$-factor is presented in Fig. 5. One can see that this simple dependence describes well the data over $b$ range from 1 to $(0.25-0.2)$ with $X=0.13$. Note, this value is three-four times as low as that for the structures with normal spectrum (see Fig. 3). Let us check how unambiguously the value of $X$ is determined for this case.

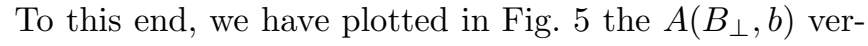
sus $b$ dependences which were calculated using two free parameters, namely $g_{\|} / g_{\perp}$ and $X$. One can see that the experimental data are equally well described with very different pairs of $X$ and $g_{\|} / g_{\perp}$.

To avoid such a large ambiguity, let us consider os- cillations of $\rho_{x x}(B)$ in a larger magnetic field, where the Zeeman splitting starts to be observed but lower than the onset of QHE. Such experimental dependence in the normal field is presented in Fig. 6 together with the curves calculated using the LK formula with different $X$ values. We assumed the Lorentz broadening of LLs with parameter $\Delta_{L}=4.5 \mathrm{meV}$ which was found from the magnetic field dependence of $\rho_{x x}(B)$ at $B<1 \mathrm{~T}$. The inset shows that the calculated curve with $X=0.13$ radically differs from the experimental curve, it does not demonstrate the Zeeman splitting of the oscillations up to $3 \mathrm{~T}$. The calculated curves with $X=0.35-0.48$ are significantly closer to the experimental dependence $\rho_{x x}(B)$, therewith the curve with $X=0.42$ practically reproduces the experimental dependence. Thus, the comparison of the data with the calculated curves in Fig. 5 and Fig. 6 gives the possibility to find unambiguously the values of $X$ and $g_{\|} / g_{\perp}$ as $0.42 \pm 0.03$ and $0.19 \pm 0.02$, respectively.

As seen from Fig. 5 some discrepancy between the $A(b) / A(1)$ data and calculated curves remains at $b<0.2$. The reasons for this discrepancy are not clear. TThey may be: (i) effect of sufficiently large in-plane component of $B$ because at $b=0.2$, the in-plane component of $B$ is about $3-4 \mathrm{~T}$, which corresponds to the magnetic length $L=(13-14) \mathrm{nm}$ so that $L$ became comparable to the width of the quantum well width $(8-9) \mathrm{nm}$. That can change the energy spectrum noticeably. (ii) a difference in the broadening (or difference in contribution to the oscillations) of different spin sub-levels; (iii) imperfect flatness of the quantum well and so on. Nevertheless, we believe that the value of $X=0.42 \pm 0.02$ corresponds to the ratio of the Zeeman splitting to the cyclotron energy in the normal magnetic field and $g$-factor anisotropy is $g_{\|} / g_{\perp}=0.19 \pm 0.02$.

The described above measurements and data treatment were carried out for all structures from Table Iover the wide electron density range. All obtained values of $X$ and $g_{\|} / g_{\perp}$ versus the electron density are summarized in Fig. 7 ,

One can see that the ratio of the Zeeman splitting to the orbital one is close to each other for both types of the structures, with the normal and inverted spectrum. This ratio decreases slightly from $X \approx 0.5$ to $\approx 0.4$, as the electron density increases from $n \approx 1 \times 10^{11} \mathrm{~cm}^{-2}$ to $5 \times 10^{11} \mathrm{~cm}^{-2}$.

The values of the $g$-factor anisotropy in the structures with normal and inverted spectra differ significantly [see Fig. [7(b)]. The values of $g_{\|} / g_{\perp}$ in the structures with the normal spectrum are in the range of $0.6-0.9$, while in the structures with inverted spectrum they are in the range of $0.05-0.015$. For both types of the structures the values of $g_{\|} / g_{\perp}$ increase with increasing electron density.

Let us compare our data with the results of previous studies. We have found only two articles [10, 11] where the Zeeman splitting was studied in the structures with $d \approx d_{c}$ and we plotted them in Fig. 7. In paper [11], the Zeeman splitting was determined for the normal magnetic field only in the structure with $d=9 \mathrm{~nm}$ at the 


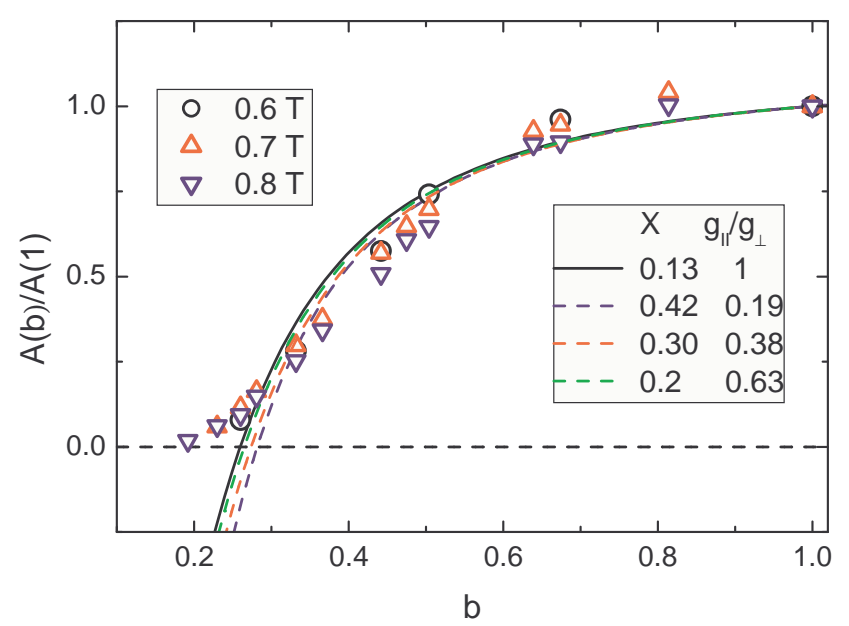

Figure 5. (Color online) The relative oscillation amplitude for structure 1023 plotted against $b$. Points are experimental data found at $B_{\perp}=0.6,0.7$, and $0.8 \mathrm{~T}$ at the electron density $n=4.6 \times 10^{11} \mathrm{~cm}^{-2}$. The lines are the dependences Eq. (3) with different pairs of the parameters $X$ and $g_{\|} / g_{\perp}$ shown in figure.

electron density $n=6.59 \times 10^{11} \mathrm{~cm}^{-2}$. The value of $X$ agrees well with our data (see Fig. 7). In Ref. 10], both the Zeeman splitting and $g$-factor anisotropy were found for the structure with $d=6.1 \mathrm{~nm}$ for the very high electron density, $n=1.46 \times 10^{12} \mathrm{~cm}^{-2}$. The value of the $g$-factor anisotropy is found to be close to our data for the structure with $d=5.6 \mathrm{~nm}$ [see Fig. [7(b)], while the ratio $g \mu_{B} B / \hbar \omega_{c}$ is significantly larger than our data: 1.26 instead of $0.4-0.5$. Such difference is unclear. One of possible reasons is role of spin-orbit interaction, which can be large for so high electron density and was not taken into account in the analysis of the data.

Now let us compare the obtained results with the theoretical ones. To find $X=g \mu_{B} B /\left(e \hbar B_{\perp} / m_{e}\right)$, the positions of the Landau levels have been calculated in framework of the 8-band $k P$ model [5]. Since there are different notations of the Landau levels in various papers, we have numbered the levels in a row, starting from unity for the lowest LL of the conduction band. The Zeeman splitting was found as the energy distance between the levels $n$ and $n+1$ with odd $n$, while the orbital splitting was found as the distance between the levels $n$ and $n+2$ [18]. The calculated $g \mu_{B} B / \hbar \omega_{c}$ versus $n$ dependences are plotted in Fig. 7(a) by solid lines. It is seen that the experimental values are slightly ower than the calculated ones for the structures with the normal and inverted spectrum. It is instructive to compare the results of the calculations performed in the framework of the 8-band $k P$ model with those obtained within the framework of the BernevigHughes-Zhang (BHZ) model [4], which is often used to analysis various effects. We have used the parameters of the BHZ model which give the dependence $E(k)$ very close to that calculated in framework of the 8-band $k P$ model. However, the Zeeman splitting in this case ap-

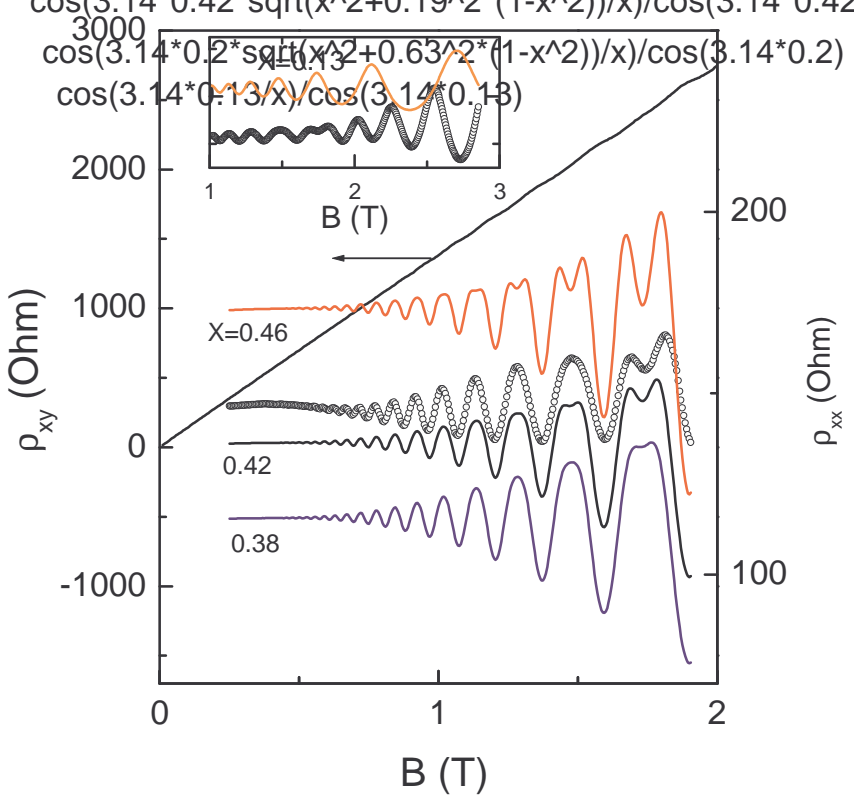

Figure 6. (Color online) The experimental dependences of $\rho_{x x}$ and $\rho_{x y}$ for structure 1023 in the normal magnetic field at $n=4.6 \times 10^{11} \mathrm{~cm}^{-2}$ for magnetic field range larger than in Fig. 4 (points). The solid lines are the calculated $\rho_{x x}$ curves with different values of $X$. These curves are shifted for clarity. The inset shows comparison of the data with the calculation with $X=0.13$ up to $B=3 \mathrm{~T}$ (see text).

pears to be $20-30$ percent larger [see dashed lines in Fig. [7(a)].

To compare the data for the $g$-factor anisotropy with the theory, one needs to know the values of $g_{\|}$together with $g_{\perp}$ calculated just above. The dependences of $g_{\|}$ on electron density were calculated using the results of the paper [19] where the energy spectrum of the $\mathrm{HgTe}$ quantum wells in the in-plane magnetic field was studied. The calculated dependences of $g_{\|} / g_{\perp}$ versus electron density are shown in Fig. 7(b). It is seen that for both types of the structures, with the normal and inverted spectrum, the theoretical values of $g_{\|} / g_{\perp}$ are small, they are close to each other, and increase with the electron density increase. The calculated values of $g_{\|} / g_{\perp}$ significantly differ from the experimental data for both types of the structures. For the structures with the inverted spectrum $\left(d>d_{c}\right)$, the experimental values are to $1.5-2$ times lower. For the structures with the normal spectrum, the difference is larger and the experimental data are to $3-4$ times higher than the calculated ones.

To discuss possible reasons for the discrepancy, let us remind the results of our previous paper [9]. We have shown (see Introduction) that for $d \approx d_{c}$ in nominally symmetric structures the top of the valence band is very strongly split by SO interaction [8]. Therewith, the SO splitting of the conduction band in the same structures does not reveal itself [9]. It is surprising that such SO splitting is observed in structures with the inverted and 

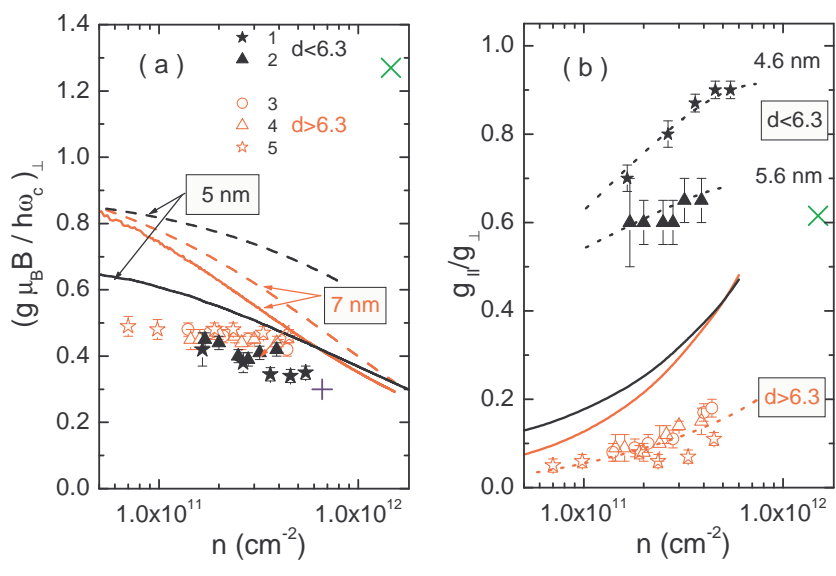

Figure 7. (Color online) The ratio of the Zeeman splitting to the orbital one at normal magnetic field (a) and $g$-factor anisotropy (b) plotted against the electron density. The solid and open symbols are the experimental data for the structures with normal and inverted spectra, respectively. The structures numbers are presented in (a). The diagonal crosses are the result of [11] and straight cross are the result of [10]. The lines are the calculated dependences for $d=5 \mathrm{~nm}$ and $d=7 \mathrm{~nm}$ (see text).

normal spectrum despite the fact that at $d<d_{c}$ and $d>d_{c}$ the conduction band is formed from different terms (see Fig. 11). It was assumed in [9] that such surprising behavior of the SO splitting is a result of the interface inversion asymmetry in the HgTe quantum well, which is not taken into account in $k P$ calculations [1 5]. We believe that the disagreement between the experimental data on $g$-factor anisotropy and calculations is also a result of the interface inversion asymmetry in the
HgTe quantum well, which is not taken into account in $k P$ calculations.

In summary, the ratio of the Zeeman splitting to the orbital one and anisotropy of the $g$-factor in the $\mathrm{HgTe}$ quantum wells both with normal and inverted spectrum have been studied experimentally within a wide electron density range. To obtain two these parameters unambiguously, we have analyzed both the tilt angle dependence of the SdH oscillations in low magnetic fields and the shape of the oscillations in moderate magnetic fields. It has been shown that the ratios of the Zeeman splitting to the orbital one are close to each other in the structures with normal and inverted spectra, these ratios decrease when the electron density increases and they are quite close to the values calculated within the $k P$ method. In contrast, the anisotropy of $g$-factor in the structures with the normal and inverted spectrum is strongly different and for both cases differ significantly from the calculated ones. We believe that such disagreement with the calculations is a result of the interface inversion asymmetry in the HgTe quantum well, which is not taken into account in the $k P$ calculations.

\section{ACKNOWLEDGMENTS}

We are grateful to S. Studenikin, V. Aleshkin, A. V. Germanenko and O.E. Raichev for useful discussions and M. Zholudev for calculations of LLs in framework of 8-band $k P$ model. The work has been supported in part by the Russian Foundation for Basic Research (Grants No. 16-02-00516 and No. 15-02-02072) and by Act 211 Government of the Russian Federation, agreement No. 02.A03.21.0006. A.V.G. and O.E.R. gratefully acknowledge financial support from the Ministry of Education and Science of the Russian Federation under Projects No. 3.571.2014/K and No. 2457.
${ }^{1}$ L. G. Gerchikov and A. Subashiev, Phys. Stat. Sol. (b) 160, 443 (1990).

2 X. C. Zhang, A. Pfeuffer-Jeschke, K. Ortner, V. Hock, H. Buhmann, C. R. Becker, and G. Landwehr, Phys. Rev. B 63, 245305 (2001).

3 E. G. Novik, A. Pfeuffer-Jeschke, T. Jungwirth, V. Latussek, C. R. Becker, G. Landwehr, H. Buhmann, and L. W. Molenkamp, Phys. Rev. B 72, 035321 (2005).

4 B. A. Bernevig, T. L. Hughes, and S.-C. Zhang, Science 314, 1757 (2006)

5 M. Zholudev, Ph.D. thesis, University Montpellier 2, France (2013).

${ }^{6}$ D. A. Kozlov, Z. D. Kvon, N. N. Mikhailov, S. A. Dvoretskii, and J. C. Portal, Pis'ma Zh. Eksp. Teor. Fiz. 93, 186 (2011), [JETP Lett. 93, 170 (2011)].

7 G. M. Minkov, A. V. Germanenko, O. E. Rut, A. A. Sherstobitov, S. A. Dvoretski, and N. N. Mikhailov, Phys. Rev. B 88, 155306 (2013)

8 G. M. Minkov, A. V. Germanenko, O. E. Rut, A. A. Sherstobitov, S. A. Dvoretski, and N. N. Mikhailov, Phys. Rev. B 89, 165311 (2014).
${ }^{9}$ G. M. Minkov, A. V. Germanenko, O. E. Rut, A. A. Sherstobitov, M. O. Nestoklon, S. A. Dvoretski, and N. N. Mikhailov, Phys. Rev. B 93, 155304 (2016).

10 M. Pakmehr, C. Bruene, H. Buhmann, L. W. Molenkamp, A. V. Stier, and B. D. McCombe, Phys. Rev. B 90, 235414 (2014).

11 X. C. Zhang, K. Ortner, A. Pfeuffer-Jeschke, C. R. Becker, and G. Landwehr, Phys. Rev. B 69, 115340 (2004).

12 N. N. Mikhailov, R. N. Smirnov, S. A. Dvoretsky, Y. G. Sidorov, V. A. Shvets, E. V. Spesivtsev, and S. V. Rykhlitski, Int. J. Nanotechnology 3, 120 (2006).

13 F. F. Fang and P. J. Stiles, Phys. Rev. 174, 823 (1968)

14 S. A. Studenikin, P. T. Coleridge, G. Yu, and P. Poole, Semicond. Sci. Technol. 20, 1103 (2005).

15 E. V. Kurganova, H. J. van Elferen, A. McCollam, L. A. Ponomarenko, K. S. Novoselov, A. Veligura, B. J. van Wees, J. C. Maan, and U. Zeitler, Phys. Rev. B 84, 121407 (2011).

16 An exception is the case when $\Delta E=0.5 \hbar \omega_{c}$, and the amplitude of the first harmonic is zero. 
17 The electron density for all structures investigated linearly depends on gate voltage $V_{g}, n\left(V_{g}\right)=n(0)+\alpha V_{g}$ and $\alpha$, within experimental error, coincides with $C /(S|e|)$, where $C$ is the capacitance measured in the same sample; $S$ is the gate area.
18 The facts that the onset of QHE is observed with even numbers and the decrease in the amplitude of the oscillations at deviation of the magnetic field from the normal to $2 \mathrm{D}$ plane show that the ratio of namely these values is determined from dependence $A(b)$.

19 O. E. Raichev, Phys. Rev. B 85, 045310 (2012). 